\title{
PRINCIPLES OF ELEMENTARY NON-WAVE THEORY OF FIELD OF ULTRASONIC TRANSDUCERS USED FOR PULSE- AMPLITUDE DEFECTOMETRY
}

\author{
V.F. Davidenko \\ Mezhevaya str. 19, ap.30, 04123, Kiev, Ukraine
}

\begin{abstract}
The elementary non-wave theory of field (ENTF) is devoted to the solution of direct and inverse problems of ultrasonic testing (UST). Solution of the direct problem of UST ENTF is to be found in energy field, and the solution of inverse problem of UST is to be found in geometric field of the transducer. Each of these fields is characterized by internal structure and external shape. The internal structure of the field is described by the equations of field lines, which are equidimensional for energy field and equisignal for the geometric field. The external shape of fields is described by energy or geometric equations of boundaries of fields, respectively. Graphical representation of the energy field with its structure and boundaries is presented in the form of ADD-diagram (amplitude-distance-defect), and that of the geometric field is presented in the form of DDA-diagram (defect-distance-amplitude). Except the symmetry of problems, fields, structures and boundaries, the asymmetry of conditions of field spreading is also important: in medium without a sound attenuation (perfect medium) and also in medium with a sound attenuation (real medium).

ENTF is based on an ordinary apparatus of higher mathematics under initial conditions, eliminating a wave nature of processes of exciting, spreading and reflection of sound pulses. Therefore, the theory is called elementary. The direct problem of UST, solved usually using $A D D$-diagram, is generally- accepted to be called defectoscopy (work in energy field), and the inverse problem, for the solution of which the DDA-diagram has not been yet used, is called defectometry (work in geometric field).
\end{abstract}

Keywords: Directivity diagram, Energy and geometric fields, Equidimensional and equisignal field lines, Cone and horn of compression, Characteristic of field braking, Specific sound attenuation, Field boundaries, Maximum range (distance) of field

\section{System of units of measurement in ENTF}

A. Physical units of measurement:

$\mathrm{r} \quad-$ distance to reflector [mm],

$\mathrm{S}-\quad-$ equivalent area of reflector $\left[\mathrm{mm}^{2}\right]$,

$\mathrm{p}=\mathrm{P} / \mathrm{P}_{\mathrm{o}} \quad-$ relative amplitude of echo-signal, dimensionless,

$\mathrm{P} \quad-$ random current amplitude of echo-signal,

$\mathrm{P}_{\mathrm{o}} \quad-$ amplitude of reference echo-signal, 
$\mathrm{d}-\quad-$ diameter of piezo-element of transducer [mm],

$\lambda-$ length of wave of ultrasonic oscillations [mm].

B. Relative units of measurement in a perfect medium:

$\mathrm{x}=\lambda \mathrm{r} / \mathrm{d}^{2} \quad-$ relative range of reflector,

$y=\sqrt{S} / \mathrm{d} \quad-$ relative equivalent dimension of reflector,

$\mathrm{n}=\mathrm{d}^{2} / \lambda^{2}-$ parameter of piezo-element, characterizing its dynamic range.

C. Scale dimensionless units of measurement in a real medium:

$\mathrm{u}=\mathrm{ngx}=\delta \mathrm{r} \quad-$ scale dimensionless range of reflector,

$\mathrm{y}=\mathrm{ngy}=\delta \sqrt{ }(\mathrm{nS}) \quad-$ scale dimensionless equivalent value of reflector,

ng $\quad-$ characteristic of field braking (scale factor) in a real medium,

$\mathrm{g}=\delta \lambda \quad-$ specific attenuation of sound (attenuation on one length of wave).

\section{Diagram of directivity (DD) of field of ultrasonic transducer}

In modern theory of UST two types of functions of directivity of fields of ultrasonic transducers are used : $2 \mathrm{~J}_{1}(\mathrm{X}) / \mathrm{X}$ - for round piezo-elements and $\sin (\mathrm{X}) / \mathrm{X}$ - for rectangular elements, where $\mathrm{X}=\mathrm{k} \cdot \mathrm{a} \cdot \sin \varphi$. However, the role, given to $\mathrm{DD}$, is rather negligible and consists only in determination of an angle of divergence of a sound beam or amplitude of echo-signal, coming from the preset direction. DD has no forming activity, influencing both the internal structure of the field, and also its external boundaries.

The known functions of DD are low-efficient, due to a difficulty of their use not only in mathematical analysis of field, but even in calculations (special Bessel tables of function are required for calculation of function $J_{1}(X)$ ). Therefore, in ENTF a successful attempt was made to derive the new function of DD, which is easily calculated, analyzed mathematically and reflects the basic properties of the forming sound field.

Derivation of the new function of DD is based on a physical-statistical model, assuming the design of a piezo-electric radiator in the form of a set of a large number of independent elementary radiators of cross-section sizes of not more than a half of length of wave of generating sound oscillations $(\lambda / 2)$. Each elementary radiator forms a field with a circular DD $\Phi(\varphi)=\cos \varphi$. At synchronous and co-phased exciting of closely-arranged elementary sound sources their fields are interacted statistically, composing into a coordinated sharp directional structure, which can be described by function $\Phi(\varphi)=(\cos \varphi)^{\pi \mathrm{n}}$. Here, the power factor $\pi \mathrm{n}$ is equal to the amount of elementary radiators of area $\lambda^{2} / 4$, arranged in a round plate of diameter $d$. And as the DD of radiation of DD of reception are equal, then under condition of radiation-reception the DD of transducer is squared and becomes equal to $\Phi^{2}(\varphi)=(\cos \varphi)^{2 \pi n}$.

This function is transformed into simpler and more convenient forms for analysis:

$$
(\cos \varphi)^{2 \pi n}=\left(1+\operatorname{tg}^{2} \varphi\right)^{-\pi n}=\left(1+a^{2} / r^{2}\right)^{-\pi n}=\left(1+n \cdot S / \pi n \cdot r^{2}\right)^{-\pi n}=\exp \left(-n \cdot S / r^{2}\right)=\exp \left(-\pi n \cdot \operatorname{tg}^{2} \varphi\right)
$$

at sufficiently high $\pi \mathrm{n}=10^{2} \ldots 10^{3}$.

The numerical comparison of the new function of DD $(\cos \varphi)^{2 \pi n}$ with known functions squared was made: $\left\{2 \mathrm{~J}_{1}(\mathrm{X}) / \mathrm{X}\right\}^{2}$ and $(\sin \mathrm{X} / \mathrm{X})^{2}$ (see Figure 1$)$, where it is seen that the new function of DD occupies, at other equal conditions, the intermediate position between two known functions. In this case, it is moved away gradually from function $\sin X / X$ in the region of small angles $\varphi$ and approaches function $2 \mathrm{~J}_{1}(\mathrm{X}) / \mathrm{X}$ in the region of large angles $\varphi$. Thus, the new function of DD possesses the uniting properties, i.e. more universal than known, and its identical forms are more analytical. Owing to this, the function of DD in the form of $\Phi^{2}(\varphi)=\exp \left(-n \cdot S / r^{2}\right)=\exp \left(-y^{2} / x^{2}\right)=$ 
$\exp \left(-\mathrm{v}^{2} / \mathrm{u}^{2}\right)$ can change them successfully, providing the feasibility of analytical modeling of structure, shape and interaction of fields of ultrasonic transducers with environment.

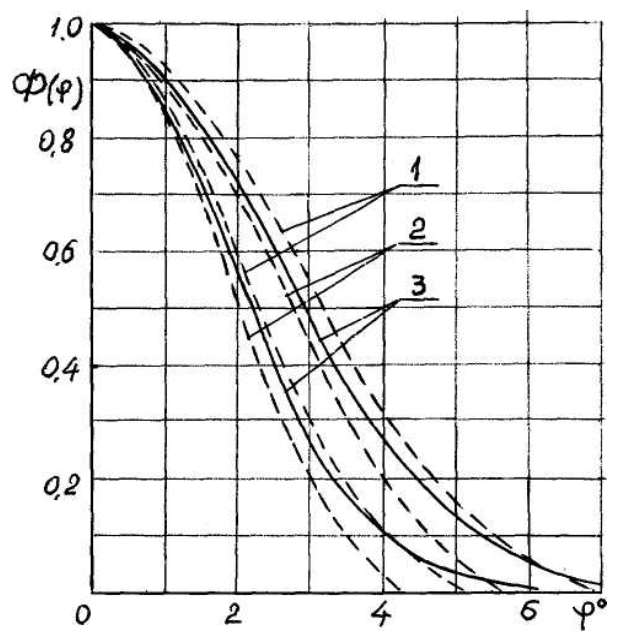

Fig.1: Comparison of different functions of DD

\section{Structure of field of transducers in a perfect medium}

In a modern theory the fields of ultrasonic transducers do not account for a differential nature of function of the field directivity when integrating the sound pressure on a removed area of the field. Therefore, there is no multiplier in the form of DD under the Kirchhoff's integral, which is introduced only after integrating to account for an angle of wave incidence on the reflector. In this connection the following integrating of pressure was made in ENTF

$$
\begin{aligned}
& \mathrm{P} / \mathrm{P}_{\mathrm{o}}=-\mathrm{ik} / 2 \pi \cdot \int_{\mathrm{S}} \mathrm{e}^{\mathrm{ikr}} \cdot(\cos \varphi / \mathrm{r}) \cdot(\cos \varphi)^{2 \pi \mathrm{n}} \cdot \mathrm{dS}=1 /(\lambda \cdot \mathrm{r}) \cdot \int_{\mathrm{S}} \exp \left(-\mathrm{n} \cdot \mathrm{S} / \mathrm{r}^{2}\right) \cdot \mathrm{dS}= \\
& =\left.\left(\mathrm{r} \cdot \lambda / \mathrm{d}^{2}\right) \cdot \exp \left(-\mathrm{n} \cdot \mathrm{S} / \mathrm{r}^{2}\right)\right|_{\mathrm{o}} ^{\mathrm{S}}=\left(\mathrm{r} \cdot \lambda / \mathrm{d}^{2}\right) \cdot\left\{1-\exp \left(-\mathrm{n} \cdot \mathrm{S} / \mathrm{r}^{2}\right)\right\}=\mathrm{x} \cdot\left\{1-\exp \left(-\mathrm{y}^{2} / \mathrm{x}^{2}\right)\right\} .
\end{aligned}
$$

The obtained result of integrating represents a relative amplitude of echo-signal $p=P / P_{o}$ from a normal disc (equivalent) reflector of a size $\mathrm{y}$, located at distance $\mathrm{x}$ from the radiator-receiver and named energy equation of field, solving the direct problem of UST and having the form

$$
\mathrm{p}=\mathrm{x} \cdot\left\{1-\exp \left(-\mathrm{y}^{2} / \mathrm{x}^{2}\right)\right\}
$$

This equation is easily transformed with respect to an equivalent size of defect, resulting in geometric equation of field allowing solution of the inverse problem of UST in the form

$$
y=+-x \cdot \sqrt{ }-\ln (1-p / x)
$$

Both obtained equations add each other in the tendency of the fullest and most comprehensive revealing of the field internal structure: one from the position of energy, while the another one from the positions of geometry. Each of these equations can be interpreted as a trajectory of special field lines : for energy field - equidimensional with parameter $\mathrm{y}=$ const., while for geometric field - equisignal with parameter $p=$ const. With the help of these field lines it is possible to represent quite in detail the specifics of a zonal structure of fields which are interdependently presented in Figures 2 and 3 in axis $x$. 


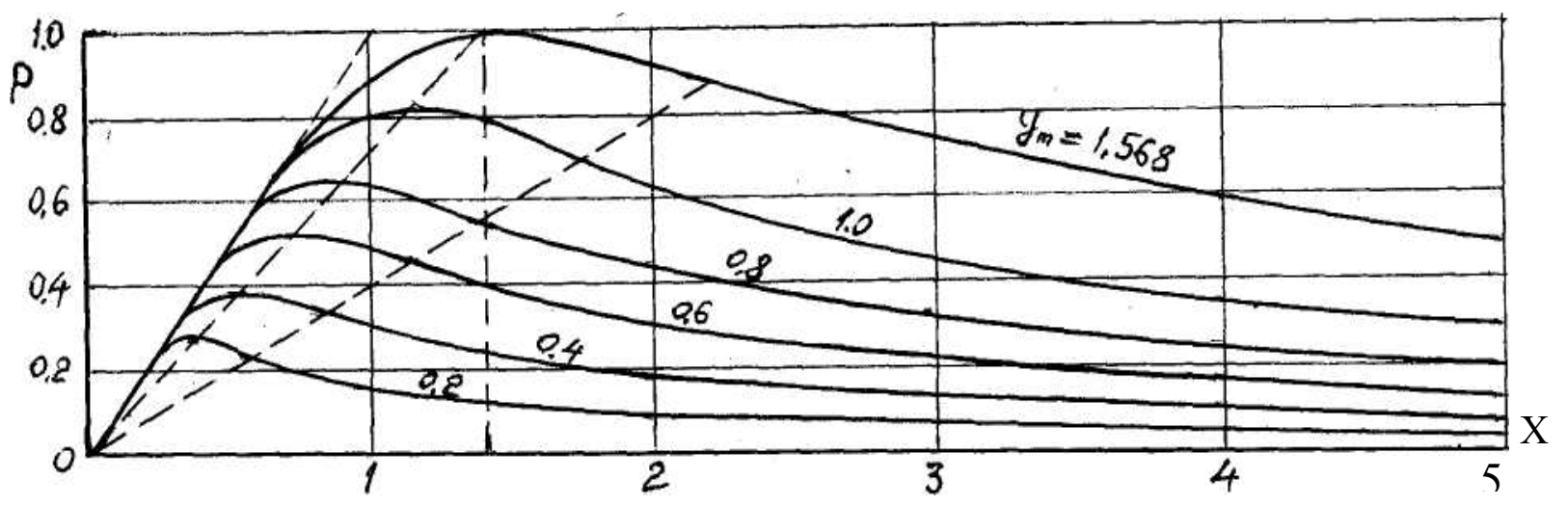

Fig. 2: Energy structure of field (ADD-diagram)

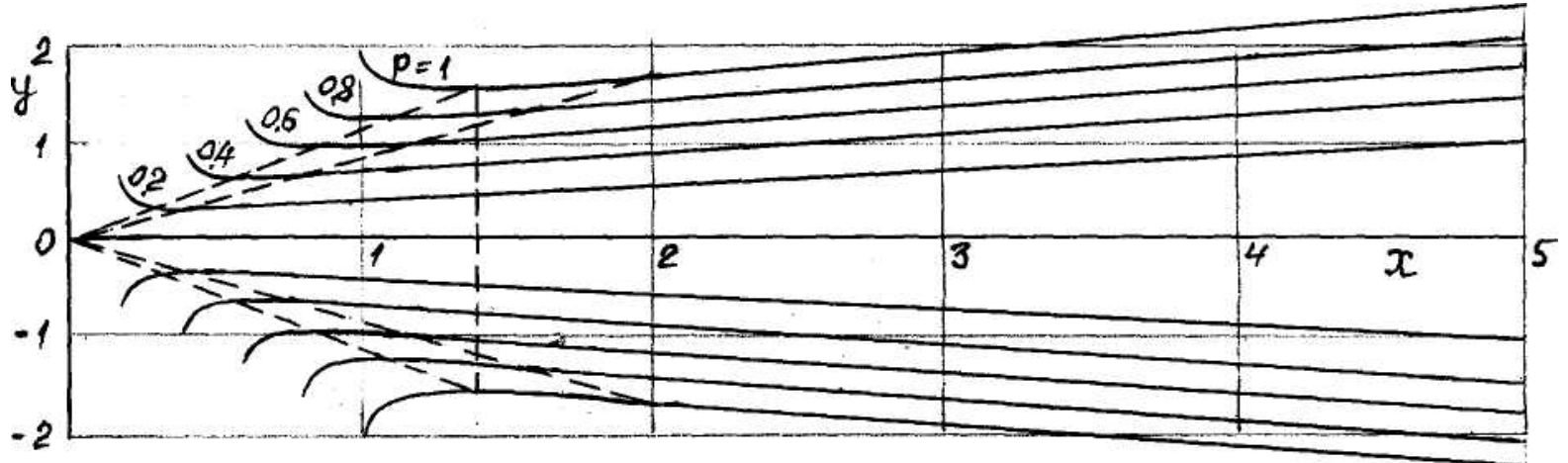

Fig. 3: Geometric structure of field (DDA-diagram)

To make analytical study of the structure of fields, the first and second derivatives are found from main variables in field length, which provide the conditions of maximum of a relative amplitude of echo-signal and minimum of equivalent size of the reflector:

$$
\begin{aligned}
& \partial \mathrm{p} / \partial \mathrm{x}=1-\left(1+2 \mathrm{y}^{2} / \mathrm{x}^{2}\right) \cdot \exp \left(-\mathrm{y}^{2} / \mathrm{x}^{2}\right)=0 \\
& \partial \partial \mathrm{y} / \partial \mathrm{x}=2 \ln \{\mathrm{x} /(\mathrm{x}-\mathrm{p})\}-\mathrm{p} /(\mathrm{x}-\mathrm{p})=0
\end{aligned}
$$

as well as conditions of inflections of equidimensional and equisignal field lines:

$$
\begin{gathered}
\partial^{2} \mathrm{p} / \partial \mathrm{x}^{2}=\left(1-2 \mathrm{y}^{2} / \mathrm{x}^{2}\right) \cdot\left(2 \mathrm{y}^{2} / \mathrm{x}^{3}\right) \cdot \exp \left(-\mathrm{y}^{2} / \mathrm{x}^{2}\right)=0 \\
\partial^{2} \mathrm{y} / \partial \mathrm{x}^{2}=\mathrm{p} /(\mathrm{x}-\mathrm{p}) \cdot\{1 /(\mathrm{x}-\mathrm{p})-2 / \mathrm{x}\}=0
\end{gathered}
$$

Graphical analytical solutions of equations (4 and 5), presented in the form of

$$
\mathrm{e}^{\mathrm{z}}=1+2 \mathrm{z}, \text { where } \mathrm{z}=\mathrm{y}^{2} / \mathrm{x}^{2} \quad \text { and }-2 \ln (1-\mathrm{z})=1 /(1 / \mathrm{z}-1) \text {, where } \mathrm{z}=\mathrm{p} / \mathrm{x} \text {, }
$$

give the following extreme solutions: $\mathrm{y}^{2}{ }_{\min } / \mathrm{x}^{2}=1.26$ and $\mathrm{p}_{\max } / \mathrm{x}=0.715$

The first extreme solution gives an equation of a generatrix of a compression cone of geometric field of the transducer in the form of line 1, presented in Figure 3.

$$
\mathrm{y}_{\min }=1.124 \cdot \mathrm{x}
$$

Substituting the physical values instead of relative values into equation (8) it is possible to find the angle of opening of compression cone from the relation $\sqrt{S}=1.77 \cdot \mathrm{a}=1.12 \cdot \lambda \cdot \mathrm{r} / \mathrm{d}$ in form 


$$
\mathrm{a}_{\min } / \mathrm{r}=\operatorname{tg} \varphi=0.63 \cdot \lambda / \mathrm{d}
$$

The obtained value of angle of compression cone opening is coincided almost with the predicted theory of diffraction ( 2 times lower than in $\sin \varphi=1.22 \cdot \lambda / \mathrm{d}$ ) taking into account that the field is studied in ENTF under the condition of radiation-reception, while in the known theories it is considered only under the condition of radiation. In spite of methodical validity of calculation of angle of opening in ENTF as compared with known procedures, the result obtained has no practical importance, probably, the same as the known results, because it refers to a perfect object, which is the field in the medium without sound attenuation. Therefore, the real structure of the field in the near zone can be studied only taking into account the attenuation that will be shown later. From the same extreme solution $\left(\mathrm{y}_{\min }=1.12 \cdot \mathrm{x}\right)$ it is possible to find also the length of the near zone using the relation $\mathrm{x}=0.89 \cdot \mathrm{y}_{\min }$ in the form

$$
\mathrm{r}_{\text {бл }}=0.89 \cdot \sqrt{ }(\mathrm{n} \cdot \mathrm{S}) .
$$

Hence, it is seen that the length of the near zone is individual for each equidimensional line of energy field because it depends not only on parameter of the transducer $n$, but also on a fixed value $S$ of the equivalent reflector. Owing to this, ENTF creates theoretical premises for the feasibility of realization of control of small sizes of reflectors near the transducer using, for example, such exotic methods as the ultrasonic microscopy. The modern theory does not provide such possibility, as well as the capabilities of conductance of UST at small depth, due to a strictly specified and rather extended near zone of the field equal to $N=a^{2} / \lambda$.

The largest length of the near-field zone can be found from the above-mentioned extreme condition $\mathrm{p}_{\mathrm{e}}=0.715 \cdot \mathrm{x}$, hence $\mathrm{x}_{\max }=1.4$, because $\mathrm{p}_{\max }=1$. After substituting physical values we obtain $r_{\max }=1.4 \cdot \mathrm{d}^{2} / \lambda$, or $r_{\max }=5.6 \cdot \mathrm{N}$ in the units of generally-accepted length of the near zone. Thus, in accordance with ENTF the length of the near-field zone can change in the range from 0 to $5.6 \cdot \mathrm{N}$, mating the far zone through a compression cone, but not through the cross-section at the distance N. Though the modern theory uses also the elementary methods of the field description, however, some wave principles are put into it a priory. They include, for example, interference model of formation of maximums of pressure in the near-field zone. The position of the last maximum is accepted as a boundary of near and far zones of the field. However, at a pulsed exciting of a piezo-element by short shock pulses this model is not valid and especially under the conditions of braking (attenuation) of sound in a real medium, that will be shown later.

Instability of formation of a plane wave in a compression cone can be explained from the point of view of ENTF that the compression cone is surrounded by a high-energy chaotic field in the form of a jacket which contacts the edges of widening plane fronts of probing pulses. Their edges have no clear limiters, undergo random actions from the side of the jacket (energy inflow) and can break, causing non-proportionally high amplitudes of echo-signals or, vice versa, dropping to a critical zero. For more visual example of effect of sound attenuation it is necessary and sufficient to compare the nature of trajectories of field lines in various media.

It is seen in Figure 2 that the equidimensional field lines are coming from a zero point of the field by a law of proportionality $\mathrm{p}=\mathrm{x}=\lambda \cdot \mathrm{r} / \mathrm{d}^{2}$, and then, by bending with convexity outside around the extreme points, locating on the generatrix of the compression cone, they begin to fall, thus changing the convexity for concavity around the points of inflection locating on straight line 2

$$
\mathrm{p}_{\text {пер }}=0.393 \cdot \mathrm{x}
$$

which was found from condition (6) in the form of $y^{2} / x^{2}=0.5$ and further from (2) in the form of $\mathrm{p}=\mathrm{x} \cdot\left(1-\mathrm{e}^{-0.5}\right)$. 
Concavity of equidimensional field lines after the point of inflections is preserved in the far zone up to infinity and has an attenuation nature

$$
\lim (\partial \mathrm{p} / \partial \mathrm{x})_{\mathrm{x} \rightarrow \infty}=1-\exp \left(-\mathrm{y}^{2} / \mathrm{x}^{2}\right)=\mathrm{y}^{2} / \mathrm{x}^{2}=0
$$

It means that the infinitely directed sound field in the attenuation medium falls nevertheless to such a level that its distance gradient is not fixed.

It is seen from Figure 3 that the equisignal field lines of the geometric field are occurred somewhere inside the chaotic jacket and tended to their minimum by bending with a convexity inside the field around points locating on the generatrix of the compression cone, then they are lifted to the field periphery, thus changing the concavity for convexity in points locating on the line of inflections:

$$
\mathrm{y}_{\text {пер }}=0.833 \cdot \mathrm{x}
$$

This straight line was obtained from (7) in the form of $\mathrm{p}=0.5 \cdot \mathrm{x}$ and further for substituting into (3) in the form of $y=x \cdot \sqrt{ } \ln 2=0.833 \cdot x$.

After the points of inflection the equisignal lines are going along the trajectory $y=\sqrt{ }(\mathrm{p} \cdot \mathrm{x})$, which means the unlimited, but slow widening of the cross-section of field with a widening gradient in infinity tending to zero.

\section{Structure of field of transducers in a real medium with sound attenuation}

Decrease in pressure in a plane sound wave spreading in a homogeneous medium occurs by the exponential law $p=p_{o} \cdot \exp (-\delta \cdot r)$, where $p_{o}$ is the pressure at the beginning of the path length $r, p$ is the pressure at the end of path $\mathrm{r}$. With account for accepted scale dimensionless variables $u$ and $\mathrm{v}$ for the medium with a sound attenuation on a double path the energy equation of the field is written in the form

$$
\mathrm{p}=\mathrm{u} \cdot\left\{1-\exp \left(-\mathrm{v}^{2} / \mathrm{u}^{2}\right)\right\} \cdot \exp (-2 \mathrm{u})
$$

Analysis for maximum of this equation shows that $\mathrm{p}_{\max }=0.5 \cdot \mathrm{e}^{-1}$. Therefore, to provide the change in a relative amplitude of echo-signals in a full dynamic range, the equation (14) should be fixed by maximum, as a result of which the energy equation of the field will have the following final form

$$
\mathrm{p} / \mathrm{p}_{\max }=\mathrm{q}=2 \mathrm{u} \cdot \exp (1-2 \mathrm{u}) \cdot\left\{1-\exp \left(-\mathrm{v}^{2} / \mathrm{u}^{2}\right)\right\}
$$

From this equation the solution of inverse problem of UST is derived in the form of the geometric equation of the field

$$
\mathrm{v}= \pm \mathrm{u} \cdot \sqrt{ }-\ln \left(1-\mathrm{q} \cdot \mathrm{e}^{2 \mathrm{u}-1} / 2 \mathrm{u}\right)
$$

Analysis of structure of energy field is started from calculation of the first derivative from a rated pressure in the field length

$$
\partial \mathrm{q} / \partial \mathrm{u}=-4 \cdot \mathrm{e}^{1-2 \mathrm{u}} \cdot\left\{1-\exp \left(-\mathrm{v}^{2} / \mathrm{u}^{2}\right)\right\}\left[\left(\mathrm{v}^{2} / \mathrm{u}^{2}\right) /\left\{\exp \left(\mathrm{v}^{2} / \mathrm{u}^{2}\right)-1\right\}+\mathrm{u}-0.5\right]=0 .
$$

Expression in square brackets is solved by a graphical analytical method using a nomogram (see Figure 4), on which an inclined line $\mathrm{y}_{1}=0.5-\mathrm{u}$ and also the family of curves, crossing it, $\mathrm{y}_{2}=\mathrm{z} /\left(\mathrm{e}^{\mathrm{z}}-\right.$ 1 ), where $\mathrm{z}=\mathrm{v}^{2} / \mathrm{u}^{2}$, are represented in $0.5 \times 0.5$ square. These curves are approximated at a 
sufficient accuracy in the ranges $0 \leq \mathrm{u} \leq 0.5$ and $0 \leq \mathrm{v} \leq 1.15$ by straight lines $\mathrm{y}_{2}=1.15 \mathrm{u} / \mathrm{v}-0.5$, coming from a pole with coordinates $(0,-0.5)$. The crossing points of the mentioned fan-like family of straight lines with an inclined line give the solution for equation (17) as $\mathrm{y}_{1}=\mathrm{y}_{2}$ or 1.15 $(\mathrm{u} / \mathrm{v})-0.5=0.5-\mathrm{u}$, which is reduced to the form

$$
\mathrm{u}=1 /(1.15 / \mathrm{v}+1)
$$

This equation has a key importance for determination of length of the field near zone in the medium with a sound attenuation. If instead of scale dimensionless variables to substitute their physical analogs, then it is possible to obtain the following expression

$$
r_{\sigma}=1 /\{1.15 / \sqrt{ }(n \cdot S)+\delta\}
$$

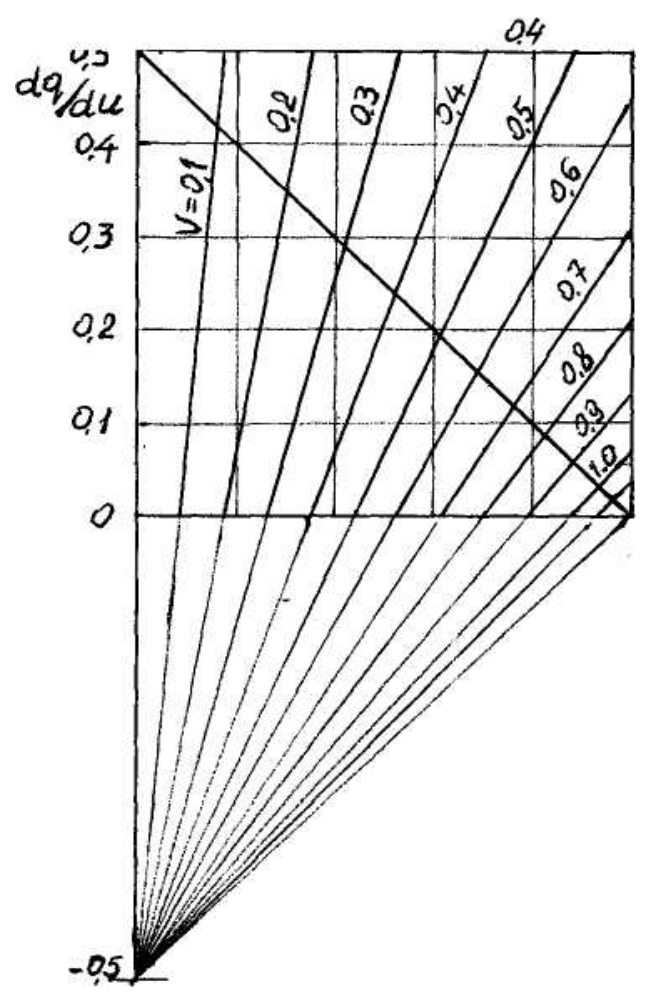

Fig. 4: A graphical analytical solution of the equation (17)

The obtained result explains visually the effect of sound attenuation on shortening of the near zone length for each equidimensional field line. If the attenuation $\delta=0$, then the length of the near zone becomes equal to the earlier obtained length for a perfect medium (10). However, the shortening of the near zone occurs not linearly, but by a more complex law, which transforms the compression cone of the field in a perfect medium into a horn of compression in a real medium. This effect can be shown by changing the angle of opening of the compression horn using the relation of its radius $a=\sqrt{ }(S / \pi)$ at the given distance $r$ from radiator to the same distance

$$
\mathrm{a} / \mathrm{r}=\operatorname{tg} \varphi=0.63 \cdot(\lambda / \mathrm{d})+0.56 \cdot(\delta \cdot \sqrt{ } \mathrm{S}) .
$$

The opening of the compression horn in the real medium at $\mathrm{S} \rightarrow 0$ occurs first by the same law as in the perfect medium, and then the law of positive feedback comes into force. The area of horn cross-section and area of the plane wave front are incremented, the resistance of medium is increased (factor $\delta \cdot \sqrt{ } \mathrm{S}$ ), angle of horn opening is increased that accelerates greatly the increase in its cross-section and leads finally to the complete flattening of the horn edge. 
The horn shape can be found as follows. Firstly, the first derivative from field section in distance is determined

$$
\partial v / \partial u=-\ln (1-z)-(0.5-u) /(1 / z-1)=0, \text { where } z=q \cdot \exp (2 u-1) / 2 u
$$

Using the graphical analytical method of analysis the solution of the following equation is found

$$
[2 u /\{q \cdot \exp (2 u-1)\}-1] \cdot \ln [1 /\{1-q \cdot \exp (2 u-1) / 2 u\}]=0.5-u
$$

which is a geometric site of maximums of relative amplitudes of echo-signals and has the form

$$
\mathrm{q}_{\mathrm{m}}=4 \mathrm{u} \cdot(1-\mathrm{u})
$$

After substituting this extreme solution into the geometric equation of the field we shall obtain the geometric equation of compression horn, having the form

$$
\mathrm{v}=\mathrm{u} \cdot \mathrm{V}-\ln \{1-2(1-\mathrm{u}) \cdot \exp (2 \mathrm{u}-1)\}
$$

The limit, to which the horn cross-section is tended, has the following solution

$$
\lim (\mathrm{v})_{\mathrm{u} \rightarrow 0,5}=0.707 \sqrt{ }\{-\ln (1-2 \mathrm{u})\}=0.707 \sqrt{ }-\ln \Delta
$$

where $\Delta$ is the relative approximation to the compression horn end (see Table 1).

Table 1

\begin{tabular}{|c|c|c|c|c|c|c|c|}
\hline$\Delta, \%$ & 0.1 & 0.2 & 0.3 & 0.4 & 0.5 & 0.7 & 1.0 \\
\hline $\mathrm{V}_{\max }$ & 1.86 & 1.76 & 1.70 & 1.66 & 1.63 & 1.57 & 1.52 \\
\hline
\end{tabular}

It is seen from Table 1, that the maximum cross-section of field in the medium with attenuation remains almost the same as that in the medium without attenuation.

Graphical presentation of energy and geometric fields of the transducers are presented in Figures 5 and 6.

The effect of sound attenuation on structure of field of the transducers can be proved by the following changes in nature of field lines in a real medium:

- Steepness of increment in amplitude of echo-signals near the transducer is almost 4 times increased $\left(\partial \mathrm{p} / \partial \mathrm{x}=1\right.$ in a perfect medium and $\partial \mathrm{q} / \partial \mathrm{u}=2 \cdot \mathrm{e}^{1-2 \mathrm{u}}$ - in a real medium).

- Steepness of increment in maximums of amplitudes of echo-signals is more than 5 times increased $(\partial \mathrm{p} / \partial \mathrm{x}=0.715$ - in a perfect medium and $\partial \mathrm{q} / \partial \mathrm{u}=4 \cdot(1-\mathrm{u})-$ in a real medium $)$.

- Steepness of increment in inflections of equidimensional field lines increases more than 3 times $(\partial \mathrm{p} / \partial \mathrm{x}=0.393-$ in a perfect medium and $\partial \mathrm{q} / \partial \mathrm{u}=1.35-$ in a real medium).

- Basic maximum of energy field is shifted closer to the transducer $\left(\mathrm{x}_{\mathrm{m}}=1.4\right.$ - in a perfect medium and $\mathrm{u}_{\mathrm{m}}=0.5-$ in a real medium).

- Gradient of decrease in amplitude of echo-signals in the perfect medium is tended to 0 infinitely, while in the real medium it is at a limited range of field, which is not more than $\mathrm{u}_{\mathrm{d}}=2.5$.

- Deformation of field in length in the medium with a sound attenuation occurs due to flattening of compression cone and its transformation with increase in the angle of opening up to $180^{\circ}$.

- Equisignal field lines of the geometric field in the medium with attenuation do not change their initial concavity for convexity, as in the perfect medium, but preserve it up to the complete bending for $90^{\circ}$. 
- Sound attenuation in the medium does not influence the diagram of directivity and, probably, the field shape due to a mutual its elimination in width and length of the field $(\mathrm{y} / \mathrm{x}=\mathrm{v} / \mathrm{u})$.

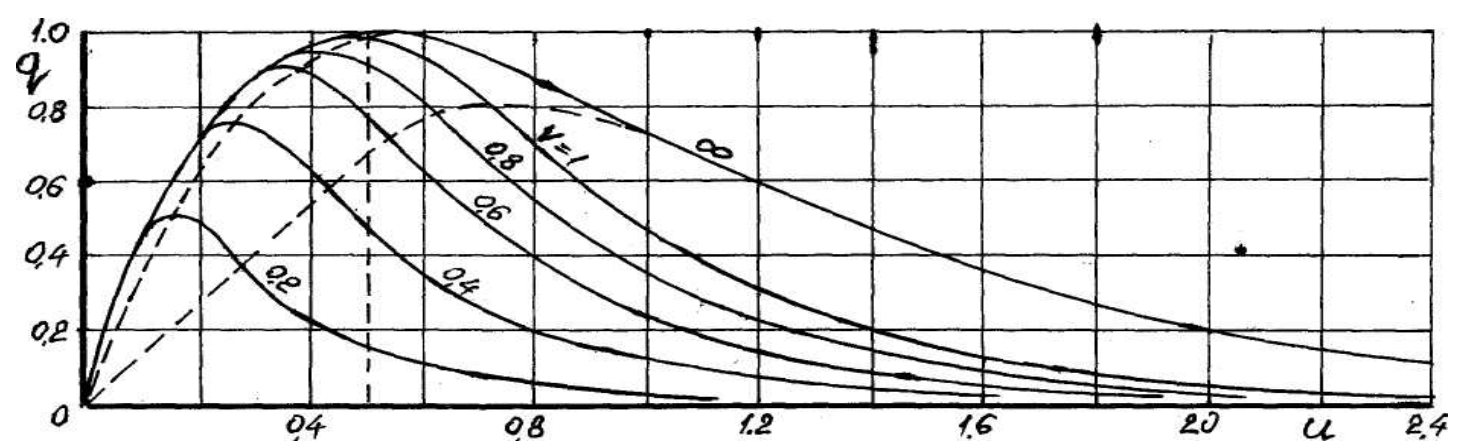

Fig. 5: Energy field of transducer in a real medium (ADD-diagram)

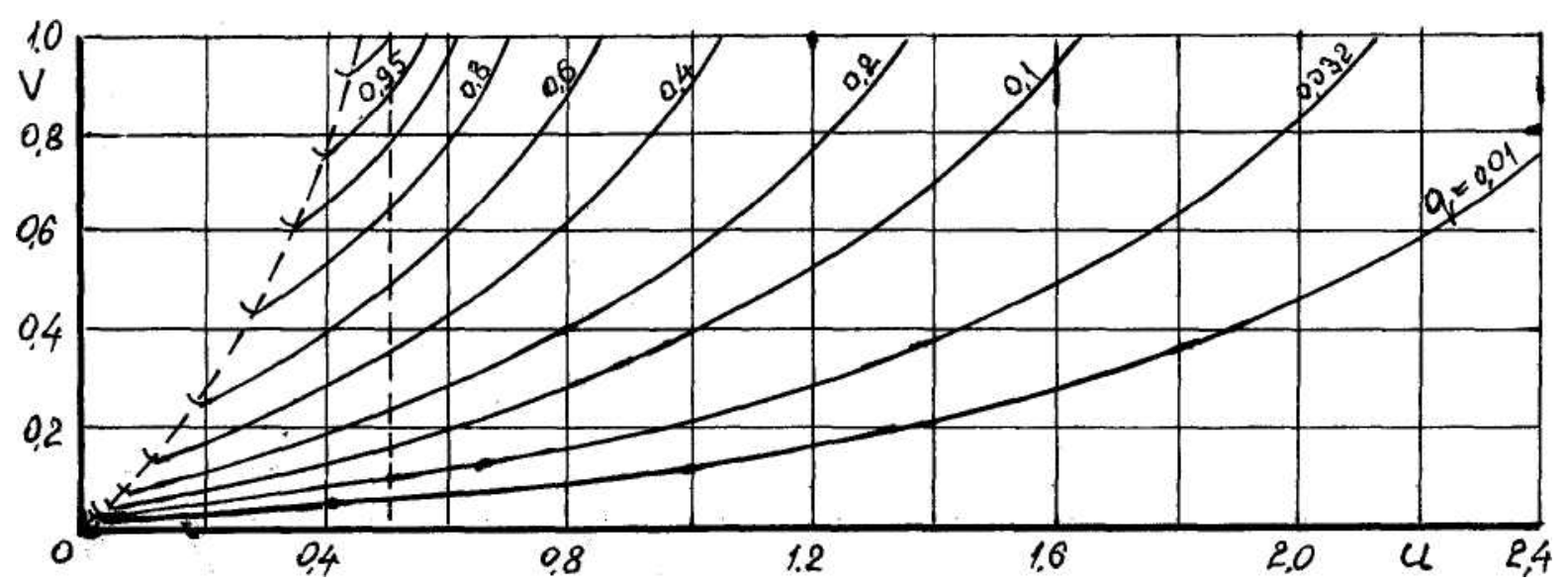

Fig. 6: Geometric field of transducer in a real medium (DDA-diagram)

\section{Boundaries of fields of ultrasonic transducers}

In the modern theory of ultrasonic transducers the forming role of DD is recognized only in the field cross-section. It is supposed that the field in length is expanded freely along the canonical isobars similar to the projector ray. This representation is not too far from data for a perfect medium obtained in ENTF. However, the equisignal field lines in the medium with a sound attenuation have a quite another nature of their trajectories and resemble the clamped bundle of straw directed by a free end towards the wind. This means that the sound field in the real medium is not finite and not conical, but has a shape predetermined by DD of the transducer not only in width, but also in length.

If to assume that the distance of penetration of the acoustic field into the real medium is proportional to the intensity of the transducer radiation in the given direction, then DD can be presented in two types coming from definition of DD as a ratio of radiation intensity in the given direction to the intensity of radiation along the radiator axis

$$
\Phi^{2}(\varphi)=\exp \left(-\mathrm{v}^{2} / \mathrm{u}^{2}\right) \approx \mathrm{u} / \mathrm{u}_{\mathrm{d}}=\mathrm{r} / \mathrm{r}_{\mathrm{d}}
$$

where $u_{d}=\delta \cdot r_{d}$ is the distance of field along a central axis (maximum distance). 
Here, the ratio of intensities of sound is changed by the ratio of proportional ranges, and the sign of approximated equality is substituted due to a change in a slant range in the preset direction for a rated range along the central axis. In connection with small angles of DD opening, the difference between the mentioned ranges is negligible.

Equation (26), obtained logically, imparts the new property to DD, which is manifested in its forming role both in transverse and in longitudinal direction of the field. Owing to this, the equation of the field shape was derived for the first time in the form of a lobe, which is accepted by intuition to represent the acoustic field (see Figure 7).

$$
\mathrm{v}= \pm \mathrm{u} \cdot \sqrt{ } \ln \left(\mathrm{u}_{\mathrm{d}} / \mathrm{u}\right)
$$

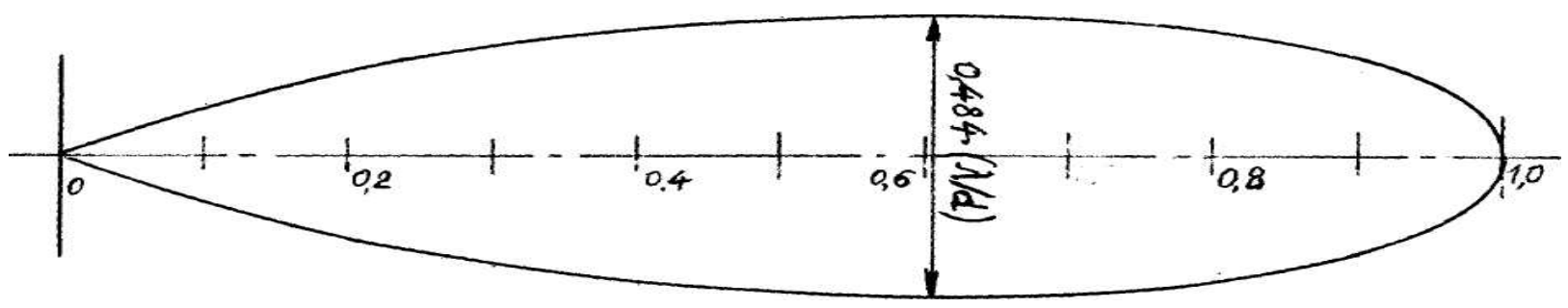

Fig. 7: Geometric boundaries of ultrasonic transducer field

Maximum width of the field is located at the distance determined from the condition

$$
\partial \mathrm{v} / \partial \mathrm{u}=\ln \left(\mathrm{u}_{\mathrm{d}} / \mathrm{u}_{\mathrm{m}}\right)-0.5=0 \text {, hence } \mathrm{u}_{\mathrm{m}} / \mathrm{u}_{\mathrm{d}}=\exp (-0.5)=0.606 \text {. }
$$

After substitution of maximum condition into formula (27) we shall find the scale maximum width of the field

$$
\mathrm{v}_{\mathrm{m}}=0.428 \cdot \mathrm{u}_{\mathrm{d}}
$$

Hence, the ratio between the maximum width of the field $b=2 a$ and its length can be expressed using physical units in the form

$$
\mathrm{b}_{\mathrm{m}} / \mathrm{r}_{\mathrm{d}}=0.483 \cdot \lambda / \mathrm{d}
$$

Thus, the fields of conventional ultrasonic transducers have ratio between the maximum length and width of field in the $12 \div 20$ range. In the modern theory the effective width of the field of ultrasonic transducers is determined coming from its conical shape at some preset distance expressed in units of length of the field near zone.

It is convenient to introduce the new rated unit of the field length in the form of $z=u / u_{d}$. Then, the geometric equation of the field will be given in a very simple expression

$$
\mathrm{v}= \pm \mathrm{u}_{\mathrm{d}} \cdot \mathrm{z} \cdot \sqrt{ } \ln (1 / \mathrm{z})
$$

which is convenient for the graphical plotting of the field (see Figure 7).

The equation of energy boundary of the field is followed from replacement of theoretical function DD for as-determined function DD in energy equation (15), i.e. $\exp \left(-v^{2} / \mathrm{u}^{2}\right)=u / u_{d}$, that leads it to the form

$$
q=2 u \cdot\left(1-u / u_{d}\right) \cdot \exp (1-2 u) .
$$


Here, the new constant $\mathrm{u}_{\mathrm{d}}$ appeared, which creates the individuality of field boundaries for each transducer by its largest length, and also by position and value of maximum of amplitude of echo-signals. The position of maximum of amplitude is determined from the condition $\partial \mathrm{q} / \partial \mathrm{u}=0$, from which the value of scale distance is equal to

$$
\mathrm{u}_{\mathrm{m}}=0.5\left\{\mathrm{u}_{\mathrm{d}}+1-\sqrt{ }\left(\mathrm{u}_{\mathrm{d}}^{2}+1\right)\right\} .
$$

After substituting this value into equation (32) the maximum amplitude of a bottom echo-signal is determined from area-unlimited reflector, normal to the axis of reflector field, having the following value

$$
\mathrm{q}_{\max }=1 / \mathrm{u}_{\mathrm{d}} \cdot\left\{\sqrt{ }\left(\mathrm{u}_{\mathrm{d}}{ }^{2}+1\right)-1\right\} \cdot \exp \left\{\sqrt{ }\left(\mathrm{u}_{\mathrm{d}}{ }^{2}+1\right)-\mathrm{u}_{\mathrm{d}}\right\}
$$

The design value of maximum of the relative amplitude of the bottom echo-signal should be taken as a coefficient of rating the individual ADD-diagrams in setting-up of sensitivity of definite transducers by bottom echo-signals in a wedge-type sample. However, for this purpose, it is necessary to determine the limiting field range of a definite transducer in the sample material. This radically new testing of the transducer is made by scanning of the sample in depth of the bottom reflecting surface by measurement of amplitude and delay of bottom echo-signals. To facilitate the procedure of sample scanning and to increase the accuracy of measurement of parameters of echo-signals, it is necessary to filter the echo-signals by a characterisitic of the highest amplitude at a fixed delay. The results obtained are substituted into the following formula

$$
\mathrm{U}_{\mathrm{d}}=\mathrm{u} /\{1-\mathrm{q} \cdot \exp (2 \mathrm{u}-1) / 2 \mathrm{u}\},
$$

which should provide the close results in different points of the sample. These results should be subjected to averaging to smooth the random fluctuations, caused by instabilities of an acoustic contact and directivity of the transducer.

Each as-tested transducer is given by ADD-diagram, differed by a rated multiplier $2 / \mathrm{q}_{\mathrm{m}}$

$$
\mathrm{q} / \mathrm{q}_{\mathrm{m}}=2 / \mathrm{q}_{\mathrm{m}} \cdot \mathrm{u} \cdot \exp (1-2 \mathrm{u}) \cdot\left\{1-\exp \left(\mathrm{v}^{2} / \mathrm{u}^{2}\right)\right\} \text {. }
$$

In practice it is convenient to use the preset estimated Table 2. With increase in a limiting distance of the field above $u_{d}>2.5$ the smaller changes occur near the maximum of the field pressure. Maximum sections of the field of transducers, corresponding to maximum equivalent reflectors, are also limited and can be determined by formula

$$
\mathrm{v}_{\mathrm{m}}=+-\mathrm{u}_{\mathrm{m}} \cdot \sqrt{ } \ln \left(\mathrm{u}_{\mathrm{d}} / \mathrm{u}_{\mathrm{m}}\right)
$$

It is seen from Table 2 that the values of the largest equivalent reflectors, real for defectometry, are in the range of $0.1<\mathrm{v}<0.6$, that corresponds approximately to 36 -fold range in area. The widening of the dynamic range of measurements of the area is possible mainly by increase in sensitivity to small defects.

Illustration of testing the transducers for distance is given in the Figure 8 in the form of a nomogram formed by the families of two types of curves. The first family of curves (1) expresses the dependence of limiting distance on the amplitude of bottom echo-signals taken from different depths of the bottom, while the second family (2) expresses the dependence of limiting distance on the values of cross-section of the field at different depths of the bottom. 
Table 2: Parameters of field for individual ADD-diagrams

\begin{tabular}{|c|c|c|c|c|c|}
\hline $\begin{array}{c}\text { Limiting } \\
\text { field } \\
\text { distance } \mathrm{u}_{\mathrm{d}}\end{array}$ & $\begin{array}{c}\text { Position of } \\
\text { max amplitude } \\
\text { of bottom } \\
\text { echo-signal } \\
\mathrm{u}_{\max }\end{array}$ & \multicolumn{2}{|c|}{$\begin{array}{c}\text { Maximum amplitude of } \\
\text { bottom echo-signal }\end{array}$} & $\begin{array}{c}\text { Maximum } \\
\text { field section } \\
\mathrm{v}_{\text {max }}\end{array}$ & $\begin{array}{c}\text { Rated } \\
\text { multiplier } \\
2 / \mathrm{q}_{\mathrm{m}}\end{array}$ \\
\cline { 3 - 5 } & $\mathrm{q}_{\text {max }}$ rel.units & $\mathrm{q}_{\mathrm{m},-\mathrm{dB}}$ & & \\
\hline 1.0 & 0.293 & 0.627 & 4.0 & 0.325 & 3.2 \\
\hline 1.2 & 0.319 & 0.673 & 3.4 & 0.367 & 3.0 \\
\hline 1.4 & 0.340 & 0.709 & 3.0 & 0.404 & 2.8 \\
\hline 1.6 & 0.357 & 0.738 & 2.6 & 0.437 & 2.7 \\
\hline 1.8 & 0.370 & 0.762 & 2.4 & 0.465 & 2.6 \\
\hline 2.0 & 0.382 & 0.783 & 2.2 & 0.492 & 2.6 \\
\hline 2.2 & 0.392 & 0.800 & 2.0 & 0.515 & 2.5 \\
\hline 2.4 & 0.400 & 0.814 & 1.8 & 0.535 & 2.5 \\
\hline 2.6 & 0.407 & 0.827 & 1.6 & 0.554 & 2.4 \\
\hline 2.8 & 0.413 & 0.838 & 1.5 & 0.571 & 2.4 \\
\hline 3.0 & 0.419 & 0.848 & 1.4 & 0.588 & 2.4 \\
\hline
\end{tabular}

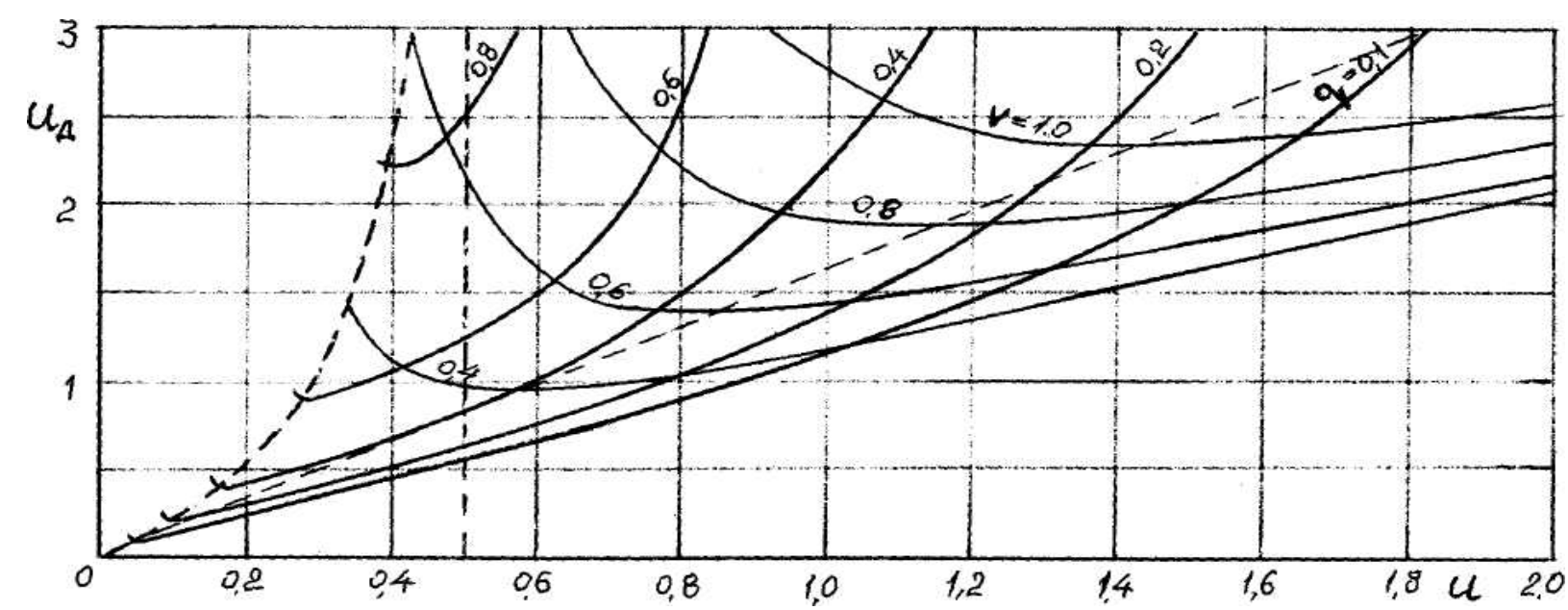

Fig. 8: Testing of transducers for limiting distance of field by parameters of bottom echosignals $(\mathrm{q}=0.1 ; 0.2 ; 0.4 ; 0.6 ; 0.8)$ and by relative cross-sections of the field $(\mathrm{v}=0.4 ; 0.6 ; 0.8 ; 1.0)$ at different depths of the bottom.

\section{Conclusions}

ENTF represents an integrated system of the new conceptions, values and relationships within the field of ultrasonic transducers, which is directed to the competition with an old system in order to search and develop the more sophisticated computer technologies of the ultrasonic testing of materials and welded joints.

\section{References}

[1] Skuchik E.: Bases of acoustic, vol.2. Translation from English. Publ. House "Mir", Moscow 1976. 542 p. 
[2] Krautkremer I. and G.: Ultrasonic testing of materials. Handbook. Translation from German, Publ. House "Metallurgia", Moscow 1991. 752 p.

[3] Shcherbinsky V.G.: Technology of ultrasonic testing of welded joints. Publ. House "Tisso", Moscow 2003. 326 p.

[4] Davidenko V.F.: New function of directivity of field of ultrasonic transducers and its practical application. Certificate about registration of the author's right for invention No.9889 of 26.04.2004. Ukraine. Ministry of Education and Science, State Department of Intellectual Property.

[5] Davidenko V.F.: Ultrasonic defectometry based on universal ADD-diagrams. Certificate about registration of the author's right for invention No. 11201 of 29.09.2004. Ukraine. Ministry of Education and Science, State Department of Intellectual Property. 